\title{
A RADIOACTIVE BEAM FACILITY USING A LARGE SUPERCONDUCTING SOLENOID
}

\author{
J.J. KOLATA, A. MORSAD, X.J. KONG and R.E. WARNER * \\ Department of Physics, University of Notre Dame, Notre Dame, IN 46556, USA \\ F.D. BECCHETTI, W.Z. LIU, D.A. ROBERTS and J.W. JÄNECKE \\ Department of Physics, University of Michigan, Ann Arbor, MI 48109, USA
}

\begin{abstract}
A facility for producing well-focussed ( $\sim 5 \mathrm{~mm}$ diameter) secondary beams of $\leq 20 \mathrm{MeV}{ }^{6} \mathrm{He},{ }^{7} \mathrm{Be}$, ${ }^{8} \mathrm{Li}$, and similar ions has been implemented. The heart of the apparatus is a superconducting solenoid operating in an asymmetric lens configuration, with large solid angle ( $\mathrm{d} \Omega \geq 100 \mathrm{msr}$ ) for the collection of reaction products. A beam of $15-\mathrm{MeV}{ }^{8} \mathrm{Li}$ ions $(\Delta E \leq 0.6 \mathrm{MeV})$ of intensity $5 \times 10^{4}$ ions/s, produced via the ${ }^{9} \mathrm{Be}\left({ }^{7} \mathrm{Li},{ }^{8} \mathrm{Li}\right){ }^{8} \mathrm{Be}$ reaction, was scattered from targets of $\mathrm{CH}_{2}, \mathrm{CD}_{2},{ }^{9} \mathrm{Be},{ }^{12} \mathrm{C},{ }^{27} \mathrm{Al}$, and ${ }^{197} \mathrm{Au}$. Elastic scattering and $\left({ }^{8} \mathrm{Li},{ }^{7} \mathrm{Li}\right)$ reaction products are unambiguously identified. Preliminary results of measurements on the ${ }^{2} \mathrm{H}\left({ }^{8} \mathrm{Li}\right.$, $\left.{ }^{9} \mathrm{Be}\right) \mathrm{n}$ reaction, as well as the status of a project to upgrade the ${ }^{8} \mathrm{Li}$ beam intensity to $\geq 1 \times 10^{6}$ ions $/ \mathrm{s}$, are reported.
\end{abstract}

\section{Introduction}

Many of the problems of contemporary nuclear astrophysics involve the determination of reaction rates for radioactive nuclides such as $\mathrm{He},{ }^{7} \mathrm{Be},{ }^{8} \mathrm{Li},{ }^{13} \mathrm{~N}$, etc. [1-3]. Such nuclei, which have meanlives ranging from seconds to days, are stable on the time scale of stellar explosions and may also play a role in primordial nucleosynthesis. In particular, considerable attention has recently been paid to the possibility that the early universe might have been rather inhomogeneous, consisting of high-density, proton-rich regions coupled with low-density, neutron-rich regions [4]. Malaney and Fowler [5] have identified a set of key reactions involving ${ }^{8} \mathrm{Li}$ which could be important for the process of nucleosynthesis in nonstandard big bangs including strong baryonic density fluctuations. The ${ }^{8} \mathrm{Li}$ nucleus is also very neutron rich and therefore of interest in more conventional nuclear spectroscopy. One might expect reactions with a $T_{z}=1$ projectile to provide unique tests of nuclear models for light nuclei. For these reasons, our work with radioactive beams has until now been confined to ${ }^{8} \mathrm{Li}$, though of course the instrument discussed below has more general utility.

The optimal study of nuclear reactions induced by a radioactive nucleus requires a device which can produce a high-intensity energy- and angle-resolved beam, focussed to a well-defined beam spot and free of contamination by other species. Devices which utilize conventional magnetic dipoles and quadrupoles $[6,7]$ usu-

* Permanent address: Physics Department, Oberlin College, Oberlin, $\mathrm{OH} 44074$, USA. ally suffer from low efficiency (due to restricted solid angle) and/or background from unwanted beams. Quadrupoles in particular are not double-focussing by themselves and therefore need to be used in doublet or triplet combinations, which usually results in a very poor aspect ratio (bore/focal length). Also, the ion orbits in dipoles and quadrupoles are not particularly simple, which can lead to complications in the design of blocking apertures and to difficulties in achieving isochronism which is important for background separation. A device which circumvents many of these problems, and appears to be well-suited to the production and focussing of beams of short-lived radioactive nuclei, is a solenoid lens [8-11]. For ion beams of energy per nucleon greater than $1 \mathrm{MeV}$, a superconducting, air-core magnet [9] can be used to advantage. The primary beam used to produce the radioactive specie travels along the solenoid axis and is therefore not deflected by the axial magnetic field, considerably simplifying the task of separating it from the reaction product of interest. The solenoid acts as a thick lens with focal length given by:

$1 / f=B_{z}^{2} L / 4(B \rho)^{2}$,

where $B \rho$ is the magnetic rigidity of the ion. An air-core magnet provides flexibility by permitting a design in which the solenoid lens is moveable, so that the system can be configured to have variable solid angle, magnification, and energy range.

A 3.5 tesla (T), $20 \mathrm{~cm}$ bore, $40 \mathrm{~cm}$ long superconducting air-core solenoid [9] has been used as a lens to collect and focus nuclear reaction products. The present configuration, which exists on a dedicated beam line from the three-stage Van de Graaff accelerator facility 


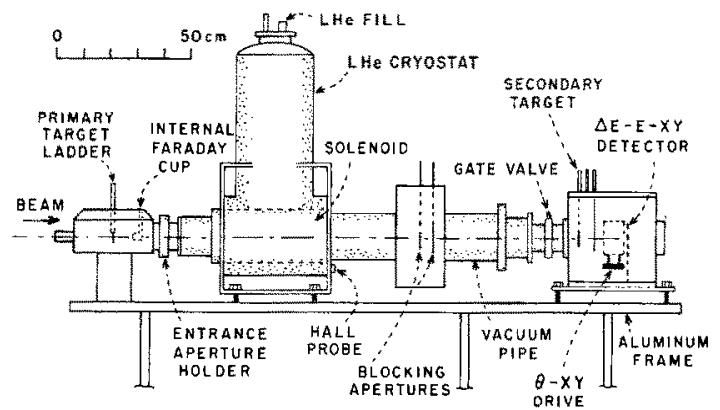

Fig. 1. Experimental apparatus for radioactive beam production.

at the University of Notre Dame, is shown in fig. 1. We have chosen an asymmetric mode of operation $\left(d_{\mathrm{obj}}=\right.$ $0.5 \mathrm{~m}, d_{\text {image }}=1.5 \mathrm{~m}$ ) which maximizes the solid angle at the expense of energy range $\left(E_{\max } / A=2 \mathrm{MeV}\right.$ per nucleon at $B_{z}=3.5 \mathrm{~T}$ ), and also results in a transverse magnification $M_{\mathrm{T}}=3$ and an angular magnification $M_{\theta}=1 / 3$. With an incident primary beam spot that is typically $2 \mathrm{~mm}$ in diameter, and a solenoid aperture opening of $3^{\circ}-11^{\circ}(\mathrm{d} \Omega=120 \mathrm{msr})$, we expect a $6 \mathrm{~mm}$ image at the secondary target, having a maximum angular divergence of about $4^{\circ}$. These parameters are quite suitable for nuclear physics experiments.

\section{Initial experiments}

For our initial study we have used beams of ${ }^{8} \mathrm{Li}$ produced via the ${ }^{9} \mathrm{Be}\left({ }^{7} \mathrm{Li},{ }^{8} \mathrm{Li}\right){ }^{8} \mathrm{Be}$ reaction at an incident ${ }^{7} \mathrm{Li}$ energy of $17 \mathrm{MeV}$. The reaction leading to the ${ }^{8} \mathrm{Be}$ ground state produces a well-defined ${ }^{8} \mathrm{Li}$ group at $E=14.3 \mathrm{MeV}$ with reasonable yield over the angular range from $3^{\circ}$ to $11^{\circ}$, which can be refocussed onto the secondary target. Since the ${ }^{8} \mathrm{Li}$ ions are more rigid than the elastically-scattered ${ }^{7} \mathrm{Li}$ beam, it is a simple matter to introduce a blocking aperture at the focal point of the latter, located in a mid-stream chamber (fig. 1). Provisions have also been made to introduce a monitor detector at this point, which produces a signal that is proportional to the primary beam current. The flight path through the solenoid system is approximately $2 \mathrm{~m}$. so that beam losses due to in-flight decay of radioactive ions with half-lives of a few hundred milliseconds (or greater) are negligible. (The half-life of ${ }^{8} \mathrm{Li}$ is $850 \mathrm{~ms}$.)

The primary ${ }^{7} \mathrm{Li}$ beam current delivered to the production target is presently limited by our ion source to 100 enA ( $3^{+}$charge state). A $12.7 \mu \mathrm{m}\left(2.3 \mathrm{mg} / \mathrm{cm}^{2}\right)^{9} \mathrm{Be}$ target then yields a secondary ${ }^{8} \mathrm{Li}$ beam of intensity $5 \times 10^{4}$ particles per second, focussed to a spot $5 \mathrm{~mm}$ in diameter and with an energy resolution that is better than $600 \mathrm{keV}$ FWHM. The beam consists of about $70 \%$ ${ }^{8} \mathrm{Li}$ ions, together with ${ }^{4,6} \mathrm{He}$ ions that have the same magnetic rigidity (fig. 2). Also visible is a group that results from correlated $\alpha$-particles arising from ${ }^{8} \mathrm{Be}$ decay, and a trace amount of ${ }^{7} \mathrm{Li}$ ions that multiplescatter within the solenoid and so avoid the primary beam block. Fortunately, the reaction $Q$-values for most ${ }^{8} \mathrm{Li}$-induced reactions are very positive, so that identification of secondary reactions involving this nucleus is generally unambiguous.

The apparatus in the secondary target chamber consists of an entrance collimator, a target ladder, and a rotatable $\Delta E-E X Y$ position sensitive counter telescope consisting of a $450 \mathrm{~mm}^{2}$ by $28 \mu \mathrm{m}$ thick planar $\mathrm{Si}$ surface barrier $\Delta E$ detector backed by a $200 \mu \mathrm{m}$ thick boron-implanted Si stopping counter. The latter detector is a $25 \mathrm{~mm}$ by $25 \mathrm{~mm}$ square with resistive cathode having up/down and left/right electrodes from which $X Y$ position can be obtained to within $\pm 1 \mathrm{~mm}$ (over much of the surface) using charge division (although there are nonlinearities near the location of the electrodes). A set of moveable apertures defines the solid angle of the telescope, which is adjustable in the range from $\mathrm{d} \Omega=3$ to $30 \mathrm{msr}$ corresponding to an angular acceptance of $\leq 13^{\circ}$. A grid was used to calibrate the $X Y$ pusition and hence the scattering angles $\theta$ and $\phi$, which are known to better than $1^{\circ}$. In a later run, a 600 $\mathrm{mm}^{2} 300 \mu \mathrm{m}$ thick is detector was also employed in the secondary reaction chamber, to act as a monitor to facilitate comparisons between runs, and to record recoil ions in coincidence with reaction products detected in the $\Delta E-E$ telescope.

Targets of $\mathrm{CH}_{2}\left(1.4 \mathrm{mg} / \mathrm{cm}^{2}\right), \mathrm{CD}_{2}\left(1.9 \mathrm{mg} / \mathrm{cm}^{2}\right)$, Be $\left(1.8 \mathrm{mg} / \mathrm{cm}^{2}\right), \mathrm{C}\left(1.3 \mathrm{mg} / \mathrm{cm}^{2}\right)$, Al $\left(1.3 \mathrm{mg} / \mathrm{cm}^{2}\right)$, and $\mathrm{Au}\left(1.0 \mathrm{mg} / \mathrm{cm}^{2}\right)$ were placed in the secondary target position in order to observe various ${ }^{8} \mathrm{Li}$-induced reactions. Elastic scattering from $\mathrm{Au}$, which has differential cross sections of the order of $10-100 \mathrm{~b} / \mathrm{sr}$, was

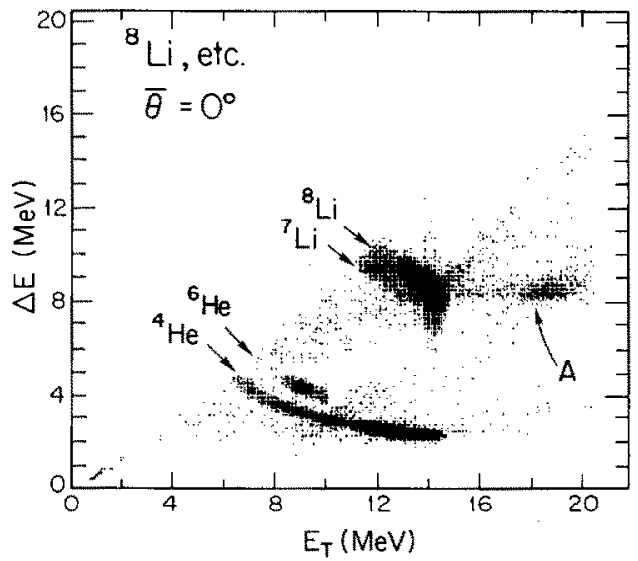

Fig. 2. A $\Delta E-E$ spectrum of the secondary ${ }^{8} \mathrm{Li}$ beam observed in-beam at the location of the secondary target. The group $A$ corresponds to ${ }^{8} \mathrm{Be}$ breakup. 


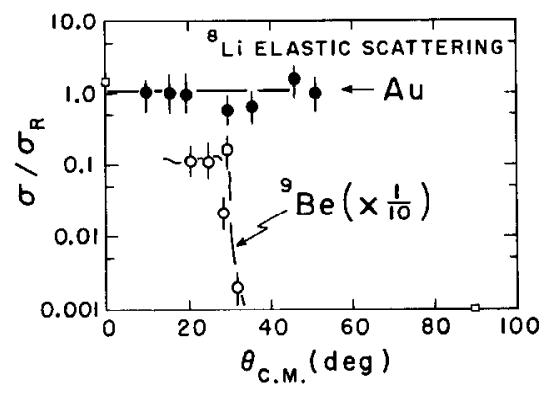

Fig. 3. Elastic scattering angular distributions observed for ${ }^{8} \mathrm{Li}$ incident on $\mathrm{Au}$ and $\mathrm{Be}$, relative to Rutherford scattering.

easily observed out to scattering angles of $45^{\circ}$ and followed the Rutherford formula as expected (fig. 3) within the constraints imposed by our $\pm 4^{\circ}$ angular resolution. The scattering from all other targets was non-Rutherford except at small angles. The angular distribution for elastic scattering from Be (fig. 3) is

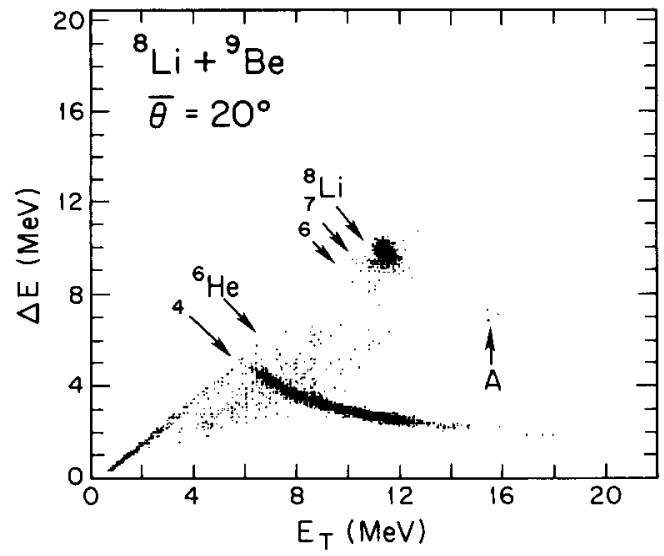

Fig. 4. A $\Delta E-E$ particle spectrum for ${ }^{8} \mathrm{Li}$ incident on a Be target. The group A corresponds to single neutron stripping leading to states in ${ }^{10} \mathrm{Be}$.

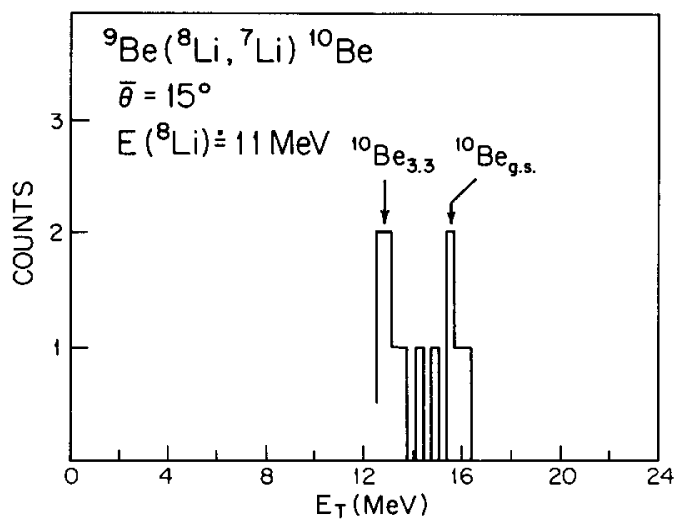

Fig. 5. Spectrum observed for the ${ }^{9} \mathrm{Be}\left({ }^{8} \mathrm{Li},{ }^{7} \mathrm{Li}\right){ }^{10} \mathrm{Be}$ reaction.

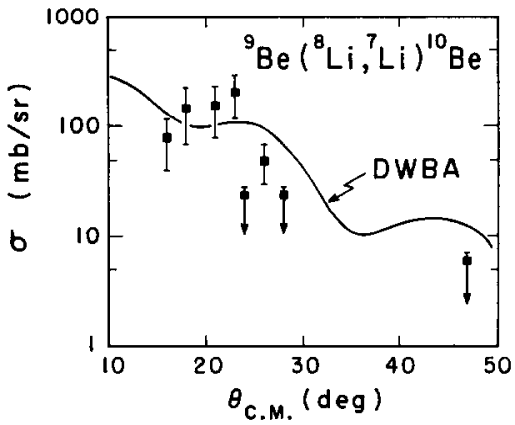

Fig. 6. Angular distribution for the particle groups in fig. 5 (summed), compared with a DWBA prediction for the same transitions.

typical and illustrates the break from Rutherford at the "grazing angle" of about $30^{\circ}$. The differential cross sections in this case are of the order of $1 \mathrm{~b} / \mathrm{sr}$ to 100 $\mathrm{mb} / \mathrm{sr}$. Such data may be used to determine the optical-model potential and thus infer the total reaction cross sections for ${ }^{8} \mathrm{Li}$.

In addition to elastic scattering, several nuclear reactions were also identified and unambiguously confirmed by target-out/target-in runs, together with the unique positive $Q$-value signal which leads to isolated groups of particles at energies well above that of the secondary ${ }^{8} \mathrm{Li}$ beam. A particularly nice example is shown in fig. 4 . The group at the location A corresponds to the ${ }^{9} \mathrm{Be}\left({ }^{8} \mathrm{Li}\right.$, $\left.{ }^{7} \mathrm{Li}\right)^{10} \mathrm{Be}$ reaction to the ground state and first-excited state of ${ }^{10} \mathrm{Be}$ (fig. 5). The differential cross sections here are typically $10-100 \mathrm{mb} / \mathrm{sr}$, and we have been able to obtain an angular distribution (fig. 6) and compare it with a distorted-wave Born approximation (DWBA) calculation. Single neutron transfer was observed for targets of ${ }^{1,2} \mathrm{H}, \mathrm{C}, \mathrm{Be}$ and $\mathrm{Al}$, with transitions to excited states being evident for the latter three targets. The experimental runs in these cases were typically $12-16 \mathrm{~h}$ in length. The reactions with ${ }^{2} \mathrm{H}$ are of astrophysical interest [5] and we have made a preliminary observation of a ${ }^{9} \mathrm{Be}$ group which may be associated with the ${ }^{2} \mathrm{H}\left({ }^{8} \mathrm{Li},{ }^{9} \mathrm{Be}\right) \mathrm{n}$ reaction. However, this signal is observed only at angles near to $15^{\circ}$ where separation from pileup and multiple processes involving the direct (secondary) beam becomes a problem. Further work will be necessary before we can unambiguously claim to have observed this interesting reaction.

\section{Conclusions}

These initial experiments have verified the feasibility of using a large solenoid to collect and focus secondary beams of short-lived radioactive nuclei that have properties suitable for nuclear physics experiments. Even at the present very low beam intensity of $5 \times 10^{4}$ par- 
ticles per second, we have been able to observe and measure angular distributions of single-neutron transfer reactions having differential cross sections in the 10-100 $\mathrm{mb} / \mathrm{sr}$ region. The next generation of experiments will utilize a modern high-intensity ion source, currently being installed at Notre Dame, that will easily deliver more than ten times the primary beam, leading to a ${ }^{8} \mathrm{Li}$ intensity of the order of $1 \times 10^{6}$ particles per second at the secondary target. With such a beam, direct measurements of differential cross sections in the few $\mathrm{mb} / \mathrm{sr}$ range can be made with reasonably high statistics. A wide range of "conventional" nuclear physics experiments with ${ }^{8} \mathrm{Li}$ beams will then be possible. We are also installing a beam buncher that will allow us to add time-of-flight information to the parameters currently being measured, to provide better background separation and identification of reaction products. Future plans include the installation of a radial electric field lens [10] to remove unwanted particles from the secondary beam and to produce a smaller beam spot size on the secondary target. Calculations show that a modest field of $\leq 50 \mathrm{kV} / \mathrm{cm}$ will produce the desired effects. Finally, the extension of the techniques described here to the production of other radioactive beams, such as ${ }^{6} \mathrm{He},{ }^{7} \mathrm{Be}$, and ${ }^{13} \mathrm{~N}$, is currently being pursued. Results on these other species could be available within the next year.

This work was supported in part by the NSF under Grant Nos. PHY84-21302 (UND) and PHY86-05907 (UM). Support was also provided by grants from the
University of Notre Dame (Jesse II. Jones Faculty Research Equipment Fund), the University of Michigan Office of Vice President for Research, and the University of Michigan College of Literature, Science and Arts.

\section{References}

[1] Proc. Workshop on Radioactive Ion Beams, ed. R.N. Boyd, IEEE Trans. Nucl. Sci. NS-30 (1983).

[2] Proc. Workshop on Prospects for Research with Radioactive Beams from Heavy Ion Accelerators, ed. J.M. Nitschke, LBL-18187 (U. Cal. - Berkeley, 1984) unpublished.

[3] Proc. Accelerated Radioactive Beams Workshop, eds. L. Buchmann and J.M.D'Auria, TRI-85-1 (TRIUMF 1985) unpublished.

[4] J.H. Applegate and C.J. Hogan, Phys. Rev. D31 (1985) 3037.

[5] R.A. Malaney and W.A. Fowler, Origin and Distribution of the Elements, ed. G.J. Mathews (World Scientific, Singapore, 1987) p. 76.

[6] R.C. Haight, G.J. Mathews, R.M. White, L.A. Aviles, and S.E. Woodward, Nucl. Instr. and Meth. 212 (1983) 245

[7] R. Bimbot et al., Z. Phys. A322 (1985) 443.

[8] J.P. Shapira et al., Nucl. Instr. and Meth. 224 (1984) 337.

[9] R.L. Stern et al., Rev. Sci. Instr. 58 (1987) 1682.

[10] W.Z. Liu, R.L. Stern, and F.D. Becchetti, Rev. Sci. Instr. 58 (1987) 220.

[11] J.M. Nitschke, Superheavy Elements, ed. M.A.K. Lodhi (Pergamon, 1978). 